LAWRENCE LIVERMORE N A T IO N A L LABORATORY
Precision damage tests of multilayer dielectric gratings for high-energy petawatt lasers

I. Jovanovic, C. G. Brown, B. C. Stuart, W. Molander, N. Nielsen, B. Wattellier, J. Britten, D. M. Pennington, C. P. J. Barty

November 10, 2004

Boulder Damage Symposium XXXVI Boulder, CO, United States September 20, 2004 through September 22, 2004 
This document was prepared as an account of work sponsored by an agency of the United States Government. Neither the United States Government nor the University of California nor any of their employees, makes any warranty, express or implied, or assumes any legal liability or responsibility for the accuracy, completeness, or usefulness of any information, apparatus, product, or process disclosed, or represents that its use would not infringe privately owned rights. Reference herein to any specific commercial product, process, or service by trade name, trademark, manufacturer, or otherwise, does not necessarily constitute or imply its endorsement, recommendation, or favoring by the United States Government or the University of California. The views and opinions of authors expressed herein do not necessarily state or reflect those of the United States Government or the University of California, and shall not be used for advertising or product endorsement purposes. 


\title{
Precision damage tests of multilayer dielectric gratings for high-energy petawatt lasers
}

\author{
Igor Jovanovic*, Curtis G. Brown, Brent C. Stuart, William Molander, Norman Nielsen, Benoit \\ Wattellier, Jerald Britten, Deanna M. Pennington, and Christopher P. J. Barty \\ National Ignition Facility Programs, Lawrence Livermore National Laboratory, \\ Mail Code L-470, 7000 East Avenue, Livermore, CA 94550, USA
}

\begin{abstract}
The next generation of high-energy petawatt (HEPW)-class lasers will utilize multilayer dielectric diffraction gratings for pulse compression due to their high efficiency and high damage threshold for picosecond pulses. We have developed a short-pulse damage test station for accurate determination of the damage threshold of the optics used on future HEPW lasers. The design and performance of the damage test laser source, based on a highly stable, high-beam-quality optical parametric chirped-pulse amplifier, is presented. Our short-pulse damage measurement methodology and results are discussed. The damage initiation is attributed to multiphoton-induced avalanche ionization, strongly dependent on the electric field enhancement in the grating groove structure and surface defects. Measurement results of the dependence of damage threshold on the pulse width, angular dependence of damage threshold of diffraction gratings, and an investigation of short-pulse conditioning effects are presented. We report record $>4 \mathrm{~J} / \mathrm{cm}^{2}$ right section surface damage thresholds obtained on multilayer dielectric diffraction gratings at $76.5^{\circ}$ incidence angles for 10 -ps pulses.
\end{abstract}

Keywords : multilayer dielectric gratings, petawatt lasers

\section{INTRODUCTION}

In the past decade, the use of chirped-pulse amplification (CPA) [1] has culminated in generation of record peak powers in excess of 1 petawatt [2] and focused intensities on the order of $10^{21} \mathrm{~W} / \mathrm{cm}^{2}$. [3] The enabling technological advance that allowed generation of petawatt peak powers from short-pulse lasers has been the aperture scaling of gold-coated diffraction gratings used in pulse compressors of CPA systems [4]. With the demonstrated apertures of $\sim 1 \mathrm{~m}^{2}$ and damage thresholds of $\sim 0.5 \mathrm{~J} / \mathrm{cm}^{2}$ for short ( $<1 \mathrm{ps}$ ) laser pulses at $1053 \mathrm{~nm}$, generation of 500-J short pulses is possible. Future applications of short-pulse lasers such as fast ignition in inertial confinement fusion [5, 6] will require much greater pulse energies (many $\mathrm{kJ}$ ) and will benefit from the use of longer pulses (2-20 ps), typically longer than the transform-limited pulse widths available from common laser gain media used to generate those pulses. Generation of such pulses, referred to as high-energy petawatt (HEPW) pulses, would require an increase of the aperture area of goldcoated diffraction gratings by an order of magnitude. This scaling requirement would result in impracticalities and possible insurmountable obstacles in the grating fabrication process, or in a necessity to tile diffraction gratings into large mosaics. [7]

The use of multilayer dielectric-coated (MLD) diffraction gratings [8] in pulse compressors of CPA systems is more promising for generation of HEPW pulses. With the previously reported damage threshold of fused silica of $\sim 5 \mathrm{~J} / \mathrm{cm}^{2}$ for 10-ps pulses at $1053 \mathrm{~nm}$ [9], optimization of the design and fabrication process of the MLD grating could result in damage thresholds which are compatible with the requirements for recompression of multi-kJ, picosecond pulses. Here we report the design, fabrication, and damage test of an MLD grating with characteristics suitable for recompression of HEPW pulses: $1780 \mathrm{~mm}^{-1}$ groove density and $76.5^{\circ}$ design incident angle allowing high spectral dispersion at $1053 \mathrm{~nm}$, high diffraction efficiency $(>96 \%)$ over the entire aperture, and highest reported damage thresholds to date $\left(4.5 \mathrm{~J} / \mathrm{cm}^{2}\right.$ for 10-ps pulses).

\footnotetext{
*Corresponding author; e-mail: jovanovic1@1lnl.gov
} 


\section{DESIGN OF MLD DIFFRACTION GRATINGS}

Optimization of grating diffraction efficiency to the maximum theoretically attainable value in order to maximize the compressor efficiency has been the traditional method of design of diffraction gratings. If the available amplified chirped-pulse energy is greater that the damage-limited energy capacity of the compressor, a more suitable merit function than diffraction efficiency alone can be utilized. This merit function is proportional to the maximum energy or peak power produced by a CPA system which utilizes the optimized grating as the final grating in the pulse compressor:

$$
M F=\eta / E_{f}^{2}
$$

where $\eta$ is the grating diffraction efficiency and $E_{f}$ is the electric field enhancement, defined as the ratio of the amplitudes of the maximum electric field in the grating structure to the incident electric field. The electric field enhancement and diffraction efficiency of the grating have been modeled using a multilayer modal method previously described. [10] The optimization of the grating performance consists of the optimization of the underlying MLD coating stack, followed by the optimization of grating groove depth and duty cycle (Fig. 1). No optimization of the grating blaze angle of the grating is performed due to the diffraction grating manufacturing process which limits the grating grooves to be orthogonal to the underlying substrate. The MLD coating stack is designed in such way that the transmitted order of light is minimized. Optimization of diffraction efficiency results in theoretical efficiency of $99.8 \%$ for a monochromatic 1053 -nm beam at an incident angle of $76.5^{\circ}$, and $E_{f} \sim 1.3$ with a duty cycle of $37 \%$ and a groove depth of $614 \mu \mathrm{m}$, giving $M F=0.59$ (Fig. 2).

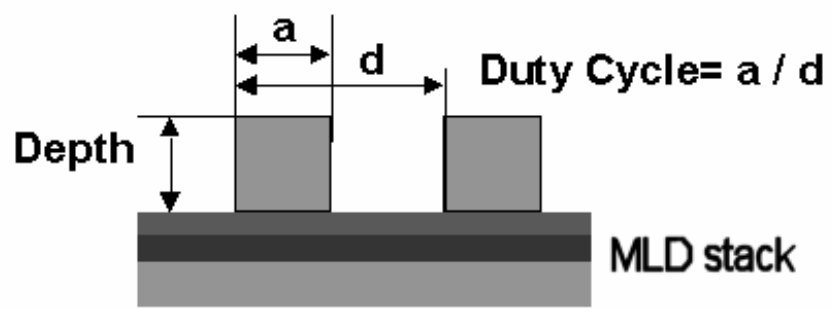

Figure 1. Schematic of an MLD grating
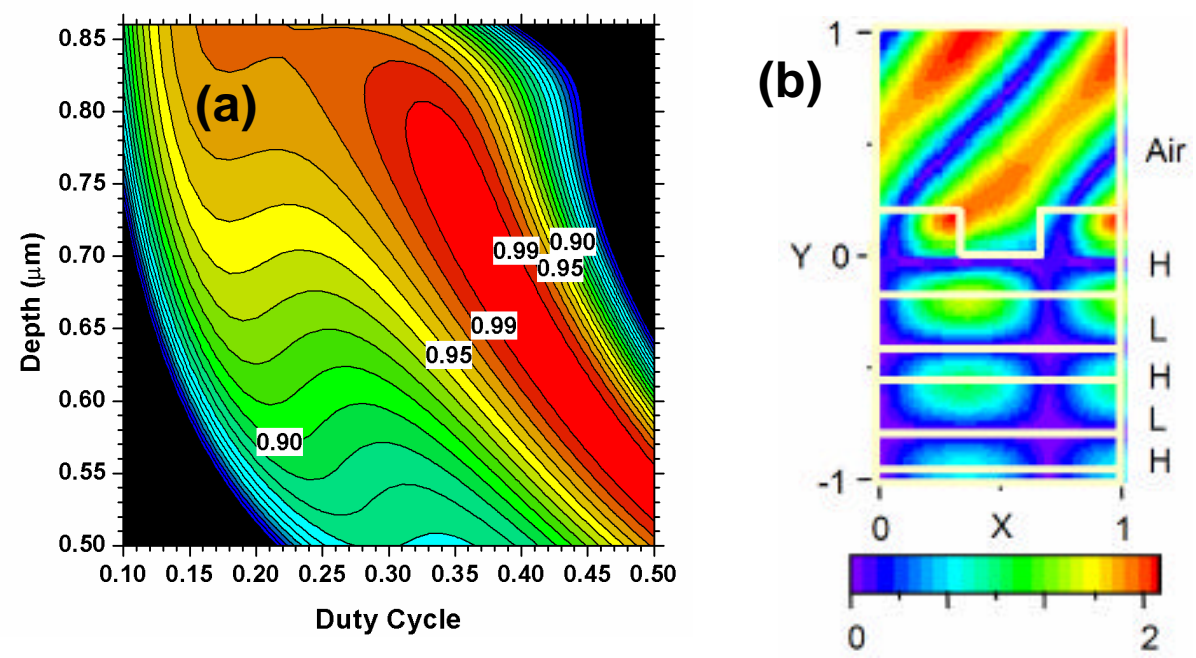

Figure 2 (a) Calculated contour maps of diffraction efficiency for a variation of depth and duty cycle on a $1780 \mathrm{~mm}^{-1}$ diffraction grating (b) distribution of electric field enhancement in one groove of a grating optimized for high diffraction efficiency (horizontal axis is in units of grating period, and the vertical axis is in arbitrary units). H and L correspond to higher and lower index layers, respectively. The electric field is maximized on the ridge edge and $E_{f} \sim 2$. 

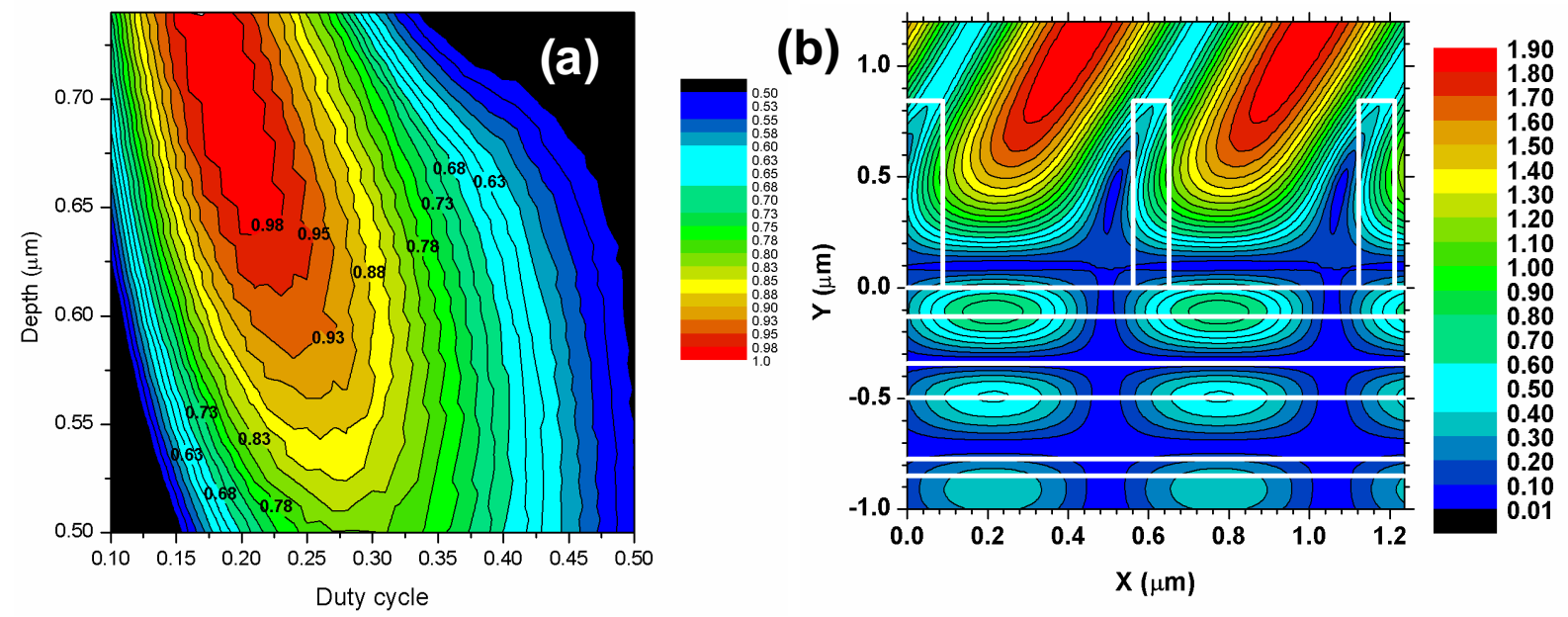

Figure 3. (a) Calculated contour maps of the merit function $M F$ for a variation of depth and duty cycle on a $1780 \mathrm{~mm}^{-1}$ diffraction grating; (b) distribution of electric field enhancement in one groove of the grating (a) optimized for maximum merit function. The electric field is maximized between two grating ridges, thus reducing the maximum electric field in contact with any part of the grating $\left(E_{f} \sim 1.07\right)$.

The optimization of $M F$, on the other hand, yields the maximum diffraction efficiency of $97.2 \%$ and $E_{f} \sim 1.07$ with a much smaller duty cycle of $16 \%$ and a groove depth of $0.749 \mu \mathrm{m}$, resulting in $M F=0.849$ (Fig. 3). An additional important consideration in designing the diffraction grating includes allowing sufficient latitude to the manufacturing process. Hence our choice of the grating design is based on the balance of maximization of our merit function $M F$ and the manufacturing process latitude. The parameters of our design point are the following: $1780 \mathrm{~mm}^{-1} \mathrm{groove}^{\mathrm{density}}$, $76.5^{\circ}$ incident angle, $16 \%$ duty cycle, and $0.740 \mu \mathrm{m}$ groove depth, resulting in $97.2 \%$ theoretical diffraction efficiency and $E_{f} \sim 1.07$, giving $M F=0.849$. The grating is on top of an MLD quarter-wave coating stack consisting of alternating layers of $\mathrm{SiO}_{2}$ and $\mathrm{HfO}_{2}$.

It is notable that the maximum electric field occurs between the grooves and the section of the grating that experiences the greatest electric field is along the ridge wall. This is in contrast to the design presented in Fig. 1(a), where the maximum electric field occurs on the ridge edge. Since the short-pulse damage is initiated at the point of maximum electric field, the design that minimizes the maximum electric field in contact with any part of the grating is predicted to be less susceptible to short-pulse laser damage and thus exhibit higher damage thresholds.

Diffraction efficiency and electric field enhancement above the grating, in the grating, and in the MLD stack for a 1780 $\mathrm{mm}^{-1}$ grating with a groove depth of $650 \mathrm{~nm}$ and an incidence angle of $76.7^{\circ}$ is shown in Fig. 4. It is apparent in from Fig. 4 that a trade-off is required between the maximum diffraction efficiency and the minimum electric field enhancement in the grating. This trade-off is represented by the merit function $M F$.

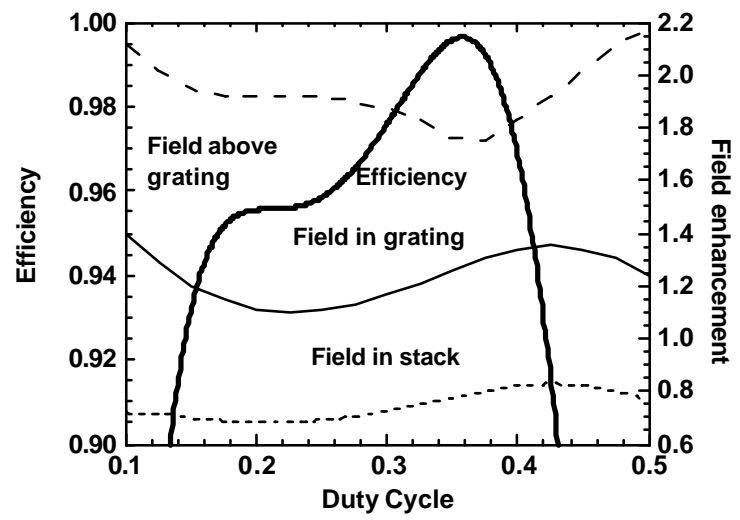

Figure 4. Efficiency and field enhancement for a $1780 \mathrm{~mm}^{-1}$ grating with a groove depth of $650 \mathrm{~nm}$ and an incidence angle of $76.7^{\circ}$ 


\section{MANUFACTURING OF MLD DIFFRACTION GRATINGS}

The holographic manufacturing method of MLD diffraction gratings imposes additional constraints on the MLD stack that is applied to the underlying substrate. The MLD stack is designed in such way that it is highly reflective for the beam at the use angle and wavelength, while it is highly transmissive for the holographic beam angle and wavelength. High accuracy of the coating deposition is required to control the grating line width over the entire aperture. Insensitivity of the grating design to coating deposition and grating line width variations is required to achieve grating uniformity. The MLD stack consists of alternating high and low index layers of $\mathrm{HfO}_{2}$ and $\mathrm{SiO}_{2}$, respectively.

$\mathrm{SiO}_{2}$ is chosen for the grating layer due to its high damage threshold. Ion beam etching is used in our process, thus requiring an etch-stop layer below the grating layer. The etch-stop layer resistant to ion beam etching is $\mathrm{Al}_{2} \mathrm{O}_{3}$.

MLD coated substrate is coated with a photoresist layer using a meniscus coating process, which is subsequently baked. Laser interference holography at $413 \mathrm{~nm}$ is used to expose the grating pattern into the photoresist layer. The optic is hard baked to increase the resistance of photoressit grating mask to the ion-beam etching process. Multi-pass reactive ion beam etching is used to etch the photoresist grating mask into the $\mathrm{SiO}_{2}$ layer. The remaining photoresist mask is removed chemically and the grating is characterized for wavefront and diffraction efficiency over the entire aperture. This method has been used to develop MLD diffraction gratings with apertures as large as $80 \times 40 \mathrm{~cm}^{2}$. [11]

\section{CHARACTERIZATION AND SHORT-PULSE DAMAGE TESTS OF MLD DIFFRACTION GRATINGS}

Scanning electron microscope (SEM) image of a manufactured MLD diffraction grating is shown in Fig. 5. This manufactured grating exhibits a greater duty cycle than our target design, reflecting the uncertainties in the grating manufacturing process. A reduction in the merit function results when the manufactured grating structure departs significantly from the design point and it illustrates the challenges of the manufacturing process. Extensive trials have resulted in a manufacturing process which can reproducibly produce structures with geometric parameters similar to the design parameters.

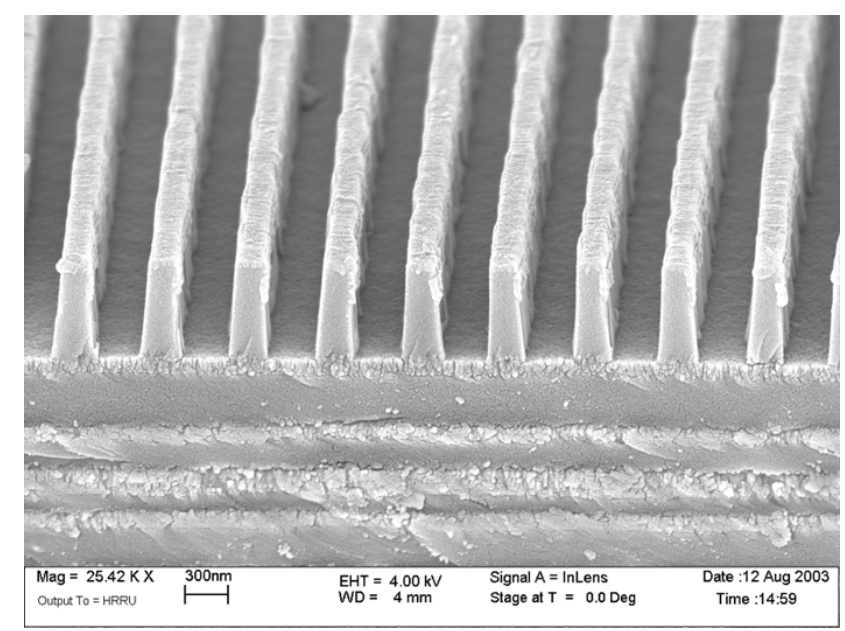

Figure 5. Scanning electron microscope image of the manufactured MLD grating

Diffraction grating efficiency across a $40 \times 80 \mathrm{~cm}^{2}$ grating aperture has been measured using a scanning photometer for a $1053 \mathrm{~nm}$ beam incident to the grating at $64.4^{\circ}$, giving a diffracted angle of $76.5^{\circ}$. The results are shown in Fig. 6. The average diffraction efficiency over the entire grating aperture is $93.5 \%$, with an rms variation of $1.9 \%$ and maximum efficiency of $98.1 \%$. This is in close agreement with the calculated diffraction efficiency. The wavefront quality of the grating has been characterized using an interferometer and determined to be ?/4 peak-to-valley, with ?/22 rms variation at $?=1064 \mathrm{~nm}$ 

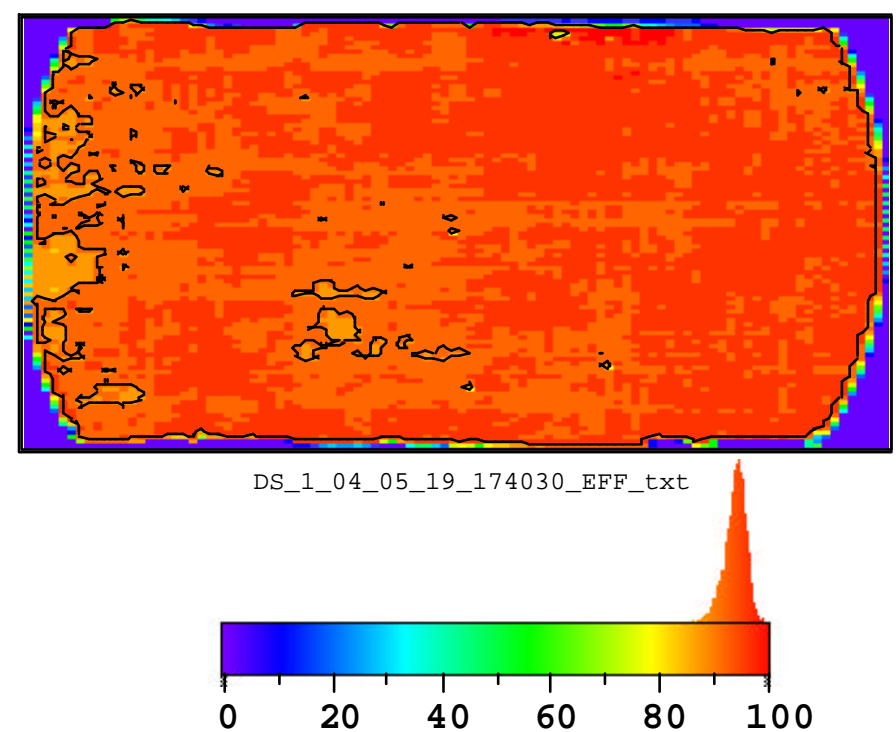

Figure 6. Results of photometer measurement of diffraction efficiency over a $40 \times 80 \mathrm{~cm}^{2}$ grating sample. The average diffraction efficiency measured is $93.5 \%$ with an rms variation of $1.9 \%$.

The damage threshold of subscale witness samples in the pulse width range of $200 \mathrm{fs}-10 \mathrm{ps}$ has been characterized using a short-pulse damage test station [12], producing $250-\mu \mathrm{m}$ diameter focal spot on the sample at a $10-\mathrm{Hz}$ repetition rate. It has been verified that no measurable self-focusing occurs in the operating range of test pulse intensities. The surface damage threshold of superpolished fused silica (SESO) at normal beam incidence has been initially measured to verify the calibration of the short-pulse damage test station. The obtained damage threshold of $5.3 \mathrm{~J} / \mathrm{cm}^{2}$ at $10 \mathrm{ps}$ is in close agreement with earlier measurements of the damage on fused silica [9]. Our damage threshold measurements on MLD diffraction gratings have been performed at the design angle of $76.5^{\circ}$ with TE polarization. Damage is observed in situ using a microscope objective and a CCD camera providing sub-10- $\mu \mathrm{m}$ resolution, as well as by measuring the diffraction efficiency for every laser shot and using an off-line Nomarsky microscope, resolving individual grating grooves. It has been experimentally determined that the sensitivity of damage detection using a Nomarsky microscope and using the in situ microscope was identical, while the sensitivity of the technique that utilizes the diffraction efficiency measurement is $\sim 10 \%$ lower. The damage threshold is defined as the fluence at which a modification of surface visible using the in situ microscope appears and grows on subsequent laser shots without modification of fluence.

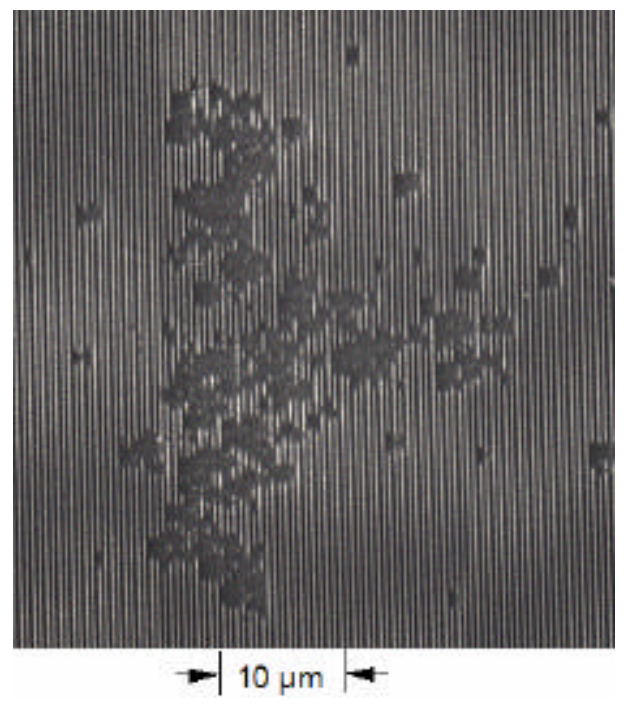

Figure 7. Typical short-pulse damage morphology for a tested MLD diffraction grating 
The damage threshold measurement is performed using two protocols: the $N: 1$ protocol corresponds to applying 600 shots at a constant fluence, while the $R: 1$ protocol corresponds to a linear increase of the fluence at a rate of $\sim 0.1 \mathrm{~J} / \mathrm{cm}^{2} / \mathrm{s}$, or $\sim 0.01 \mathrm{~J} / \mathrm{cm}^{2} / \mathrm{shot}$. The damage fluence is reported normal to the beam axis. Our $N: 1$ measurement results in $3.2 \mathrm{~J} / \mathrm{cm}^{2}$ damage threshold, while the R:1 measurement results in $4.6 \mathrm{~J} / \mathrm{cm}^{2}$ damage threshold. An SEM photograph of the typical damage morphology observed is shown in Fig. 7.

The departure of the damage threshold of tested MLD gratings from the design predicted damage threshold is the result of several factors. The departure of the manufactured geometry from the design geometry leads to different field enhancement. Post-processing steps, particularly photoresist removal has a significant effect on the damage threshold when residual photoresist exists in the grating grooves. Finally, defects in the MLD coatings such as inclusions or crazing and simple contamination can decrease the damage threshold.

Some laser conditioning effects are observed as the $R: 1$ damage threshold is $\sim 40 \%$ greater than the $N: 1$ damage threshold for this sample. Conditioning effects are attributed to the ejection of defects and contaminants embedded on or in the vicinity of the grating surface, which typically occurs at fluences lower than the damage threshold of an ideal grating structure. Contamination can arise from residues of the grating manufacturing process and can be reduced by use of additional post-processing steps such as acid bath etching. The difference between $N: 1$ and $R: 1$ damage thresholds suggests the advantage of the gradual increase of fluence on the grating in a CPA system to maximize the available energy of the system. The difference between the $R: 1$ and $N: 1$ damage threshold has been studied systematically on a large number of damage test samples and the results are shown in Fig. 8. It is noted that the R:1 measurement results in higher damage threshold for a majority of samples we tested.

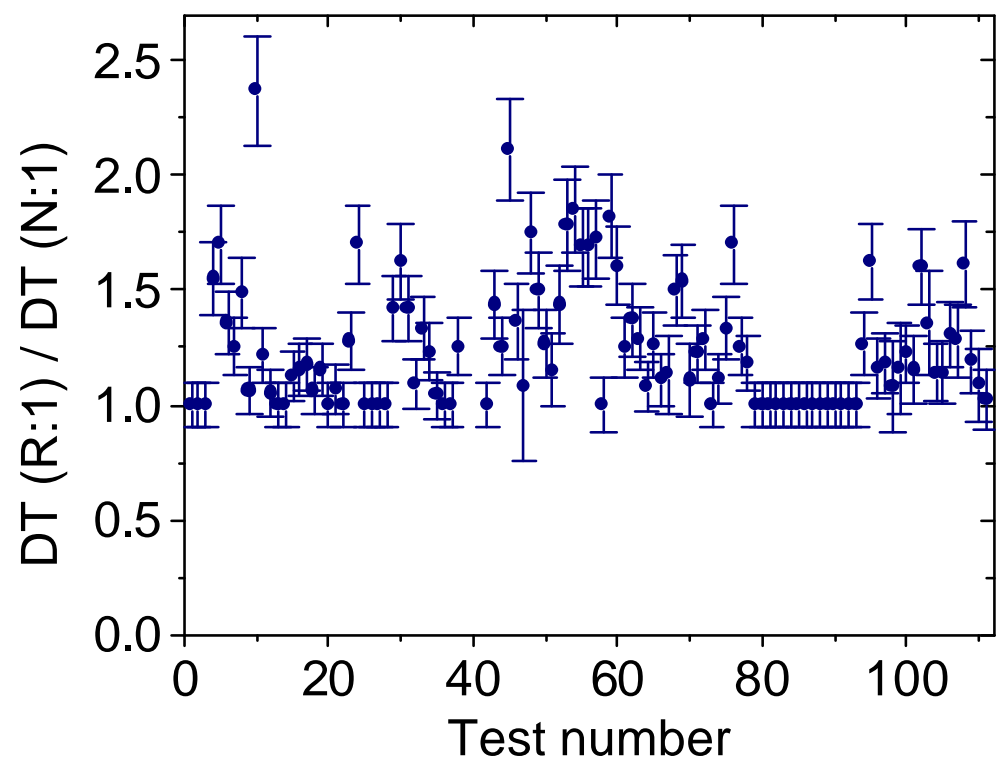

Figure 8. Ratio of R:1 and N:1 measured damage thresholds for $>100$ tests reveals the existence of conditioning effects for majority of the tested samples

Pulse width dependence of damage threshold has been evaluated by scanning the pulse width between the minimum available pulse width of $200 \mathrm{fs}$ to $20 \mathrm{ps}$. The results are shown in Fig. 9, also for a $1780 \mathrm{~mm}^{-1} \mathrm{grating}$ sample. The measured pulse width dependence resembles a power law, with the least-square fit proportional to $\mathrm{t}^{0.29}$, where $\mathrm{t}$ is the FWHM pulse width. This is in good agreement with previous measurements performed on dielectric and the short-pulse damage model which attributes the damage to the multiphoton-induced avalanche ionization. [9]. 


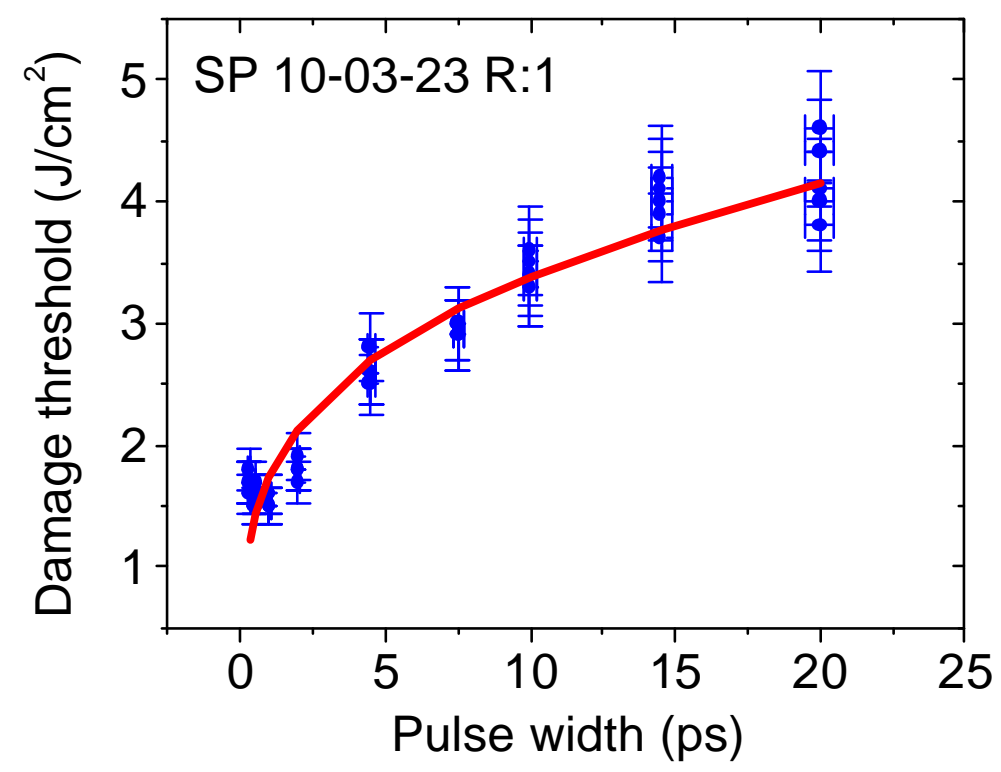

Figure 9. Pulse width dependence of damage threshold. Best power fit indicates that the damage threshold scales with $\tau^{0.29}$, where $\tau$ is the pulse width.

Dependence of damage threshold on the use angle at a pulse width of 10 ps has been measured around the design angle of $76.5^{\circ}$ and is shown in Fig. 10. The results reveal the effects of electric field enhancement. If the effects of field enhancement were not present, the damage threshold would result in a simple 1/cos law for damage threshold. The fit of the measured data to the 1/cos law are also shown in Fig. 10.

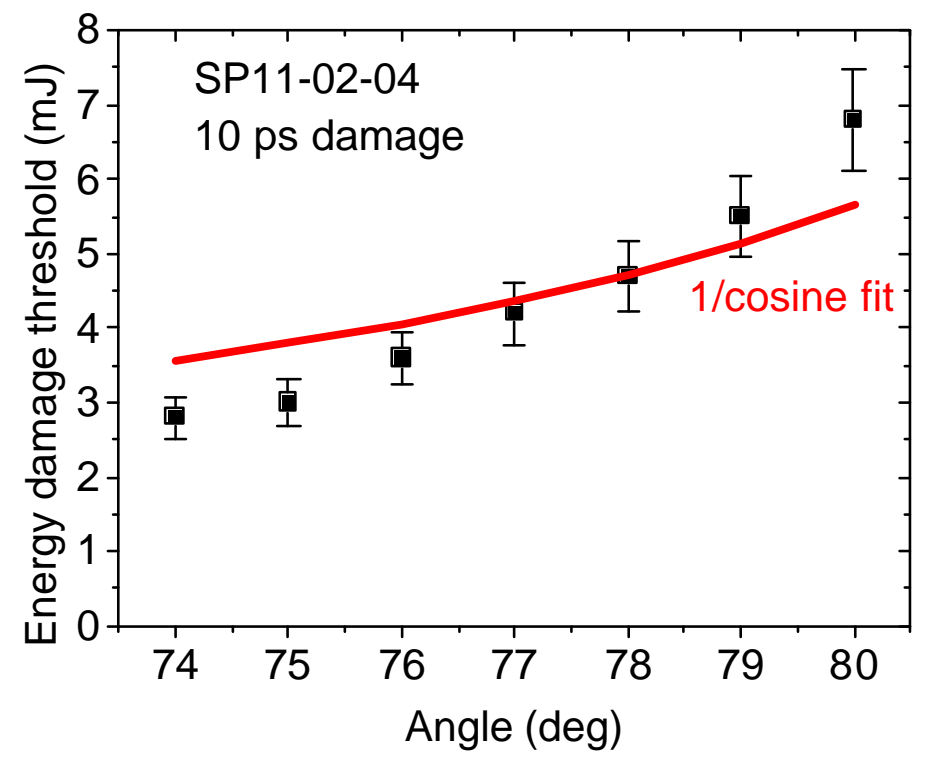

Figure 10. Dependence of the damage threshold on the use angle. The solid line represents the best $1 / \cos ($ angle) fit to the experimental data. 


\section{CONCLUSION}

In conclusion, we have demonstrated the highest damage threshold multilayer dielectric diffraction gratings to date for use in CPA systems. The design of the grating has been optimized to maximize the merit function, enabling maximum pulse energy from a pulse compressor that utilizes this design for its final grating. Dependence of the damage threshold on the pulse width suggests $\sim 2.5$ times increase of the available energy from the CPA system when the pulse width is increased from 500 fs to $10 \mathrm{ps}$. The measured damage threshold of $\sim 4.6 \mathrm{~J} / \mathrm{cm}^{2}$ at $10 \mathrm{ps}$ represents an improvement of nearly one order of magnitude over the existing gold-coated diffraction gratings. This characteristic, along with the high spectral dispersion at $1053 \mathrm{~nm}$ resulting from the $1780 \mathrm{~mm}^{-1}$ groove density and $76.5^{\circ}$ design incident angle makes this grating ideally suited for generation of HEPW pulses when scaled to $\sim 1-\mathrm{m}^{2}$ aperture.

\section{ACKNOWLEDGEMENTS}

The authors wish to acknowledge support of the Lawrence Livermore National Laboratory's LDRD Progra m. This work was performed under the auspices of the U. S. Department of Energy by the University of California, Lawrence Livermore National Laboratory under Contract No. W-7405-Eng-48.

\section{REFERENCES}

1. D. Strickland and G. Mourou, "Compression of amplified chirped optical pulses," Opt. Commun. 56, 219-221 (1985).

2. M. D. Perry, D. Pennington, B. C. Stuart, G. Tiethohl, J. A. Britten, C. Brown, S. Herman, B. Golick, M. Kartz, J. Miller, H. T. Powell, M. Vergino, and V. Yanovsky, "Petawatt laser pulses," Opt. Lett. 24, 160-162 (1999).

3. J. D. Bonlie, F. Patterson, D. Price, B. White, and P. Springer, "Production of $>10^{21} \mathrm{~W} / \mathrm{cm}^{2}$ from a large-aperture Ti:sapphire laser system,” Appl. Phys. B 70, 155-160 (2000).

4. R. D. Boyd, J. A. Britten, D. E. Decker, B. W. Shore, B. C. Stuart, M. D. Perry, and L. Li, "High-efficiency metallic diffraction gratings for laser applications," Appl. Opt. 34, 1697-1706 (1995).

5. M. Tabak, J. Hammer, M. E. Glinsky, W. L. Kruer, S. C. Wilks, J. Woodworth, E. M. Campbell, M. D. Perry, and R. J. Mason, "Ignition and high gain with ultrapowerful lasers," Phys. Plasmas 1, 1626-1634 (1994).

6. R. Kodama, "Nuclear fusion - Fast heating scalable to laser fusion ignition", Nature 418, 933-934 (2002).

7. T.J. Kessler, J. Bunkenburg, H. Huang, A. Kozlov, and D.D. Meyerhofer, "Demonstration of coherent addition of multiple gratings for high energy chirped-pulse-amplified lasers", Opt. Lett. 29, 635-637 (2004).

8. M. D. Perry, R. D. Boyd, J. A. Britten, D. Decker, B. W. Shore, C. Shannon, and E. Shults, "High-efficiency multilayer dielectric diffraction gratings," Opt. Lett. 20, 940-942 (1995).

9. B. C. Stuart, M. D. Feit, S. Herman, A. M. Rubenchik, B. W. Shore, and M. D. Perry, "Nanosecond-to-femtosecond laser-induced breakdown in dielectrics," Phys. Rev. B 53, 1749-1761 (1996).

10. L. Li, "Multilayer Modal Method for Diffraction Gratings of Arbitrary Profile, Depth, and Permittivity," J. Opt. Soc. Am. A 10, 2581-2591 (1993).

11. J. A. Britten, "Demonstration of Meter-Scale, High Laser Damage Multilayer Dielectric Diffraction Gratings for HEPW Pulse Generation," $1^{\text {st }}$ International Conference on Ultrahigh Intensity Lasers, Lake Tahoe, California, October 3-7, 2004.

12. I. Jovanovic, C. Brown, B. Wattellier, N. Nielsen, W. Molander, B. Stuart, D. Pennington, and C. P. J. Barty, "Precision short-pulse damage test station utilizing optical parametric chirped-pulse amplification", Rev. Sci. Instrum., in press (2004). 